\title{
ECO-FRIENDLY ENZYMATIC PRODUCTION OF ORGANIC ACIDS
}

\author{
Abhay kumar \& Vignesh $\mathbf{M}^{*}$ \\ Prajakt chemical, Road No: 7, Plot No: 705, GIDC, Sachin, Surat, Gujarat, India. \\ geneticvicky@gmail.com ${ }^{1}$ \\ *Correspondence: \\ Vignesh Marimuthu \\ Prajakt chemical, Road No: 7, Plot No: 705, GIDC, Sachin, Surat, Gujarat, India \\ Tel: +916382664483 \\ E-mail: geneticvicky@gmail.com
}

\begin{abstract}
The bio catalytic synthesis of the organic acid is one of the most promising biopolymers due to the fact that produced from the non-toxic renewable resource of food or grain waste. Therefore, organic acid is an ecofriendly yield with best features for the industrial usage (Non-toxicity). The biological based productions are efficient in providing products that are a replacement for petrochemicals and bio functionality advantages. The commercial enzymatic treatment as hydrolysis of the substrate to help in reduces the production schedule and cost effective. In this article focus on different bioconversion of anaerobic digestion along with enzymatic hydrolysis- obtained as food or grain waste from current organic acid production process. We explained the recent different organic acid production over on an enzymatic hydrolysis and fermentation technology.
\end{abstract}

Key words: Organic acids, Fermentation, Eco-friendly enzymes hydrolysis.

\section{Introduction}

A consecutive of the environment to replace the traditional chemical techniques with the biological source of commercial enzyme can helps to form of organic acid has exposed fugal and bacteria actual striking cell mass. The fungal based commercial enzyme from use production of less molecular weight organic compounds present in all organism and having more carboxyl groups (Habeet al., 2015). Organic acid have potential of role in natural ecology and industrial applications as food additives, and leather, textile applications. They are full produced biodegradable polymers and replaced petroleum and synthetic chemicals reactions.

These topic concerns about the commercial enzyme based to production of organic acids via anaerobic oxidation of biomass Level of fermentation in the process quality or which comprise prospective for future enzymatic development of organic acid. 


\section{Why enzymatic production of organic acid?}

Bio absorbable and degradable of polymeric organic acid considered to alternative to the improvement and development of improvement and development of numerous applications in industrial usage. The awareness and ecofriendly with since the twenty-first century has purpose to alternative chemical based acids around the world focusing on the bio based production of organic acid via enzymatic anaerobic fermentation digestions. The objective of the synthesis of organic acid via enzymatic process to help of cost effective and while production end of the effluent to control the environmental conditions.

\section{Anaerobic digestion into organic acids and classification}

Microbiology of anaerobic digestion of organic waste is process which pretreatment of commercial enzymes and involves different bacteria species, such as hydrolytic and acid forming, acetogenic and methanogenic produce the organic acids.

\subsection{Hydrolysis}

The hydrolysis processes of the polymerized, mostly in soluble organic compounds, as carbohydrate, proteins, fat are decomposed into soluble monomers and dimers that as monosaccharaides, amino acids and fatty acids. This stage of the methanogenesis passes through extracellular enzymes from the group of hydrolysases (amylase, protease and lipase) produced by appropriate strains of hydrolytic bacteria. Hydrolysis of hardly decomposable polymers, that is, cellulose and cellulo-cottons is considered to be a stage which limits the rate of wastes digestion. The rate of hydrolysis is depending parameter such as $\mathrm{pH}$, production of enzyme and absorption on the particles of wastes to digestion process. Simultaneous scarification and fermentation process carried out L-lactic acid produced from pretreatment of enzymes acremonium cellualse to hydrolysis corncob (substrate) (Miuraet al., 2004). The lactic acid fermentation from agriculture residues such as soybean straw, through enzymatic (cellulase) hydrolysis maximum rate $51.22 \%$ of cellulose and hemicellulose were pretreated (Xuet al., 2007). The study involves the production of short chain organic acids from kitchen waste as anaerobic conversion of organic fraction of domestic solids wastes to given the three types of organic acids as lactic acid (65-85\%), acetic acid (10-30\%), propionic acid (5-10\%), and butyric acid (5-20\%) (Lohet al., 1999). enzymatic hydrolysis carried out by commercial enzyme and bacteria the group of relative anaerobes genus like streptococcus and enterobacterium.

\subsection{Acidogenesis (Acidification Phase)}

During this process, acidifying bacteria convert substrate of organic food waste to short chain of organic acids. This bacterium is act as water soluble chemical substance hydrolysis and produce organic acid, alcohols, aldehydes, carbon di oxides and hydrogen. This process is working as two pathways to produce the high level of hydrogen ion to recognize the acidogenesis process. The acid phase bacteria belonging to facultative anaerobes use an occasionally introduce, create anaerobic favorable conditions for the development of obligatory anaerobes of the following genera: Pseudomonas, clostridium, micrococcus, or Flavobacterium and bacillus. 


\subsection{Actinogenesis}

Methnobacterium propionicum, syntrophomonas, syntropphobacter convert the acid phase products into acetates and hydrogen molecules, produced by methanogeneic bacteria, which species one of the Methanobacterium subodans account for decomposition of propionic acid to acetic acid (Schinket al., 1997). In acetogenesis process, approximately $25 \%$ of acetates are formed and approximately $11 \%$ of hydrogen is produced in the organic wastes degradations (Glassneret al., 1992). Methanogenesis is only involves to produce the methane gas and co-polymeric products.

\section{Types of organic acid and enzyme based anaerobic digestion}

\subsection{Citric acid}

Citric acid (2-hydroxy-propane-1, 2, 3-tricarboxylic acid) is a derivative of Latin word citrus. Citric acid other names as tricaboxylic acid, colorless, and freely soluble in water (Molecular weight (MW): $210.14 \mathrm{Da}$ ). These acids significantly have special features as decomposable, sustainable, cost effective, and multi-use chemical for wetting, buffering, cleaning, and dissolving citric acid most common metabolic product.

\subsubsection{Citric acid production}

\subsubsection{Synthesis of citric acid developments}

A fungi and yeast microbes suitable and addition with bacteria by using the mutation and fermentation competitive with selective strain can be used for production process (Soccolet al., 2006). aspergillus niger, A. aculeatus, A. awamori and yeast as saccharomyces lipolytica, candida tropicalis, c. oleophila, C. guilliermondii can be forming a citric acid in considerable amount. amoung the bacterial species, i.e bacillus lichiniformis, Arthobacter paraffinens and cornybacterium species, anciently use for production by using many raw material including enzymes as substrate with the percentage yield of 27-28\% sugar consumes by the microbial strains. The microbial production of citric acid is considered along with catalysis of enzymes. those enzymes to regulate the tircarboxylic acid (TCA) cycle is responsible for production of citric acid and quantatively production of acids depending upon the enzymes and cofactor elements concentrations. Aeration of growth through help carbon di oxide is important as a substrate for pyruvate carboxylase that replenishes the supply of oxaloacetic acid for citric synthase. The catalytic enzymes to help of produce carbon di oxide by pyruvate decarboxylase, excessive aeration to some losses (Kubiceket al., 1980). Although ammonia and glucose supplementary to catabolism of citric acid production issue by alpha- ketoglutrate dehydrogenase, inhibit the synthesis of citric acid. Other then, concern of citric acid production are of interest to other enzymes includes: hexokinase, invertase, glucose oxidase, phosphofructokinase and pentose phosphate pathway (Maxet al., 2010) (Khanet al., 2017).

\subsection{Acetic acid}

Vinegar main component as acetic acids, is a trational; acidic acid condiment which is originally cooking. Acetic acid bacteria (AAB) are gram negative obligate aerobic, which is oxidise with anaerobic and aerobic condition to form acetic acid. AAB is grown by semi 
continuous biotransformation process. this process mainly have two stages like alcohol to acetaldehyde and acetaldehyde to acetic acid and have two enzymatic catalytic reaction as ethanol oxidized to acetaldehyde by a pyrroloquinoline quinine dependent alcohol dehydrogenase (ADH) and then acetaldehyde further oxidizing to acetic acid by an aldehyde dehydrogenease (ALDH) (Yakushiet al., 2010) Those enzymatic activity of ADH and ALDH are ideally regulatory sites to enhance the vinegar productivity by AAB.

\subsubsection{Acetic acid production}

The three method used for the production of major group of acetic acid are the Orleans method (slow method), the Trickling (quick), submerged fermentation. The initial two methods is a very ancient method.

\subsubsection{The Orleans method}

While the wine making to left in open vats became converted to vinegar by AAB entering the atmosphere. The open field process of vinegar has disadvantages as acetic acid not reduces the $\mathrm{pH}$ and many of the microorganisms survive with $\mathrm{AAB}$.

\subsubsection{The Trickling generators method}

The wood tanks constructer to use produce acetic acid, and occasionally of stainless steel, a false bottom support, wood shaving and separates them from the collection chamber which one fifth of the total capacity of the generator. Here the spray mechanism distributes of heat generator via a pump circulate the acetic acid-alcohol mixture to circulate from the reservoirs, which over packing much same of the generator whereas trickling filter functions in waste water treatment (Fig.1). Cooling water in the heat exchanger is used to control the temperature with monitoring using temperature knob and air force is through in the falsebottom up to the setup. Compulsory a three parameter is necessary to check: 1 . circulation of the mash, 2. flow of the cooling water via heat exchanger, 3. circulation of aeration level in the tank. The both of orlean and trickling method had not use the enzymes for fast addition for growth of acetic acid.

\subsubsection{Enzymatic Submerged fermentation method}

Submerged fermentation defined as fermentation in the presence of excess water. These types of fermentation as a pretreatment of enzymes into large scale production of organic acids on the recent industrial continue operations. The food waste FW or grains waste have been treated to produce the necessary output. Initially, enzymatic hydrolysis of FW is conducted in a protease at $60^{\circ} \mathrm{C}$, alpha amylase keep at $90-95^{\circ} \mathrm{C}$ and Amyloglucosidase keep at $55-60^{\circ} \mathrm{C}$. After hydrolyzed the FW should be centrifuged and supernatants to be use acetic acid elaboration through the two fermentation process includes: alcoholic fermentation (anaerobic) and acetic fermentation (aerobics) condition.

\subsubsection{Alcoholic fermentation (Anaerobic)}

The FW hydrolyzed supernatant is inoculated with yeast genus in the production fermenter. The alcoholic fermentation carried out in taking capacity at $30{ }^{\circ} \mathrm{C}$ for $48 \mathrm{~h}$ at stationary phase (Pazuchet al., 2018). 


\subsubsection{Acetic fermentation (aerobic)}

The production ethanol percentage corrected 5\%, a recommended $70 \%$ ethanol is add $10 \%$ of stronger acetic acid add ratio proportion in the 1.0:1.5, subsequently carbon and nitrogen source is added to the acetic acid fermentation in a bench top fermenter at $30^{\circ} \mathrm{C}, 0.25 \mathrm{VVM}$ aeration and strring at $300 \mathrm{rpm}$ (Pazuchet al., 2019).

\subsubsection{Current developments acetic acid synthesis}

Acetic acid is naturally produced by anaerobic hemoacetogens and aerobic aectobacter species, the metabolic engineering has been widely used in strain in the conventional bed batch fermentation process to produce the acetic and butyric acid (Yanget al., 2013).DiazRicciet al.,( 1991) described the glucose metabolism of E. coli strain bearing mutation abolishing both acetyl phosphotranferase (PTA) and acetokinase (ACK) activities was studied anaerobic and aerobic conditions among with common cloning vector pU19, and mutation carrying a plasmid bearing 'pet' operon that carrying mutation showed that low specific growth rate and glucose uptake rates relative to parent wild type of K-12 applied to the fermentation that significantly enhance acetic acid production volume and while that produced low amount of lactic acid and formic acid no accumulation and lower secretion of the ethanol and succinate. The batch type of rector metabolism of using commercial immobilized penicillinamidase (E.C.3.5.1.11) to growth as a tertazole -1- acetic acid (TzAA esters) with saturated alcohol is reported (Kostadinovet al., 1992). Even Tryptophan is converted to indole - 3- acetamate and indole -3- acetic acid by enzymes from pseudomonas savastanoi for used enzymes from kernels of zea mays sweet corns (Michalczuket al., 1982) (Rekoslavskayaet al., 1994).

\subsection{Succinic acid}

Succinic acid is also known as 1, 2- ethanedicarboxylic acid, amber acid, obtained from varies form of natural source of ester. Usual application of succinic acid include detergent, food additives, cosmetics, pigments, cement additives, soldering fluxes, and leather chemical intermediates.

\subsubsection{Production and metabolic anaerobic pathway}

Succinic acid is a tetra carbon dicarboxylic acid production is one of the anaerobic fermentation products of metabolism (Leeet al., 2006). Intermediate of several biochemical pathways, succinic acid produced by many microorganisms. The co-product of ethanol fermentation by the yeast saccharomyces cerevisiae to challenges of produce the succinic acid at commercial scale and potentially for the chemical market (Samuelovet al., 1991). Anaerobiospirillum succiniproducens was the first anaerobic bacteria isolated and shown to produce succinic acid (Guettleret al., 1996). The pathway of reductive TCA cycle in the gram negative bacteria was biochemically revealed and the fermentation by- products, acetic acid, formic acid, were elimination near classical method (Leeet al., 2006). The notable utilization of 5,6 carbon sugar can breakdown and hydrolysate to form the pyruvate, simultaneously, which changes to oxaloacetate, its convert to the formation of malate and end of the yield succinic acid production through an anaerobic digestion (Fig.2). Even though gene knock out technology was used to found the whole genome sequence from the 
succinic acid producing rumen Bactria Mannheimia succiniproducens carried out the anaerobic metabolism and amoung the three different co2-fixing metabolic reactions catalyzed by phospoenolpyruvate (PEP) carboxykinase, PEP carboxylase, and mallic enzyme was most important for the anaerobic growth of succnic acid (Leeet al., 2006).

\section{Conclusion}

Organic acids are beneficial chemicals for future biorefining applications. Such applications involve the conversion of low cost renewable resource to platform chemicals by fermentation and further derivative to large-volume chemicals through conventional catalytic routes. Nowadays, current knowledge shows that organic acids not only act as carbon metabolisms as intermediates, somehow nutrient deficiencies, metal tolerance and plant-microbes interactions operating at the root-soil interphase. Even though organic acids are toxic to many microorganisms such as E.coli and which are combined with beneficial microorganisms the effect and act as antibiotic in the animal nutrition. Investigated ecofriendly enzymatic pretreatment are numerous beneficial effects in the production of organic acids. . In this article focused on different bioconversion of anaerobic digestion along with enzymatic hydrolysis- obtained as food or grain waste from current organic acid production process. These of enzymatic treatment including improve the low cost production, and while, waste water treatment could be reduce the Chemical oxygen demand (COD) and Biological oxygen demand BOD in the process. The food waste and organic waste will be consume produce the better organic acid than chemical via production.

\section{ACKNOWLEDGMENTS}

The present research was supported by the research fund of Prajakt chemical in 2022.

\section{Reference}

[1] H. Habe, S. Sato, T. Morita, T. Fukuoka, K. Kirimura and D. Kitamoto, "Bacterial production of short-chain organic acids and trehalose from levulinic acid: a potential cellulose-derived building block as a feedstock for microbial production", Bioresource technology, 177, (2015), pp.381-386.

[2] C. R. Soccol, L.P. Vandenberghe, C. Rodrigues and A. Pandey, "New perspectives for citric acid production and application”, Food Technology \& Biotechnology, (2006), 44(2).

[3] C.P. Kubicek, O.Zehentgruber, H. El-Kalak and M. Röhr, "Regulation of citric acid production by oxygen: effect of dissolved oxygen tension on adenylate levels and respiration in Aspergillus niger", European journal of applied microbiology and biotechnology, 9(2),(1980), pp.101-115.

[4] B. Max, J.M. Salgado, N. Rodríguez, S.Cortés, A.Converti and J.M.Domínguez, "Biotechnological production of citric acid", Brazilian journal of Microbiology, 41(4), (2010), pp.862-875.

[5] I. Khan, S. Qayyum, F. Maqbool, A. Hayat and M.S. Farooqui, "Microbial organic acids production”, biosynthetic mechanism and applications-Mini review (2017). 
[6] C.W. Loh, A. Fakhru'l-Razi, M.A. Hassan and M.I.A. Karim, "Production of organic acids from kitchen wastes”, Artificial Cells, Blood Substitutes, and Biotechnology, 27(5-6), (1999), pp.455-459.

[7] S.T. Yang, M. Yu, W.L. Chang and I.C. Tang, "Anaerobic fermentations for the production of acetic and butyric acids", Bioprocessing technologies in biorefinery for sustainable production of fuels, chemicals, and polymers, (2013), pp.351-374.

[8] J.C. Diaz-Ricci, L. Regan and J.E. Bailey, "Effect of alteration of the acetic acid synthesis pathway on the fermentation pattern of Escherichia coli", Biotechnology and bioengineering, 38(11), (1991), pp.1318-1324.

[9] M. Kostadinov, A. Nikolov, N. Tsoneva and N. Petkov, "New tetrazole-1-acetic acid esters for enzymatic synthesis of cefazolin", Applied biochemistry and biotechnology, 33(3), (1992), pp.177-182.

[10] L. Michalczuk and J.R. Chisnell, "Enzymatic synthesis of 5-3H-indole-3-acetic acid and 53H-indole-3-acetyl-myo-inositol from 5-3H-L-tryptophan”, Journal of Labelled Compounds and Radiopharmaceuticals, 19(1), (1982), pp.121-128.

[11] N.I. Rekoslavskaya and R.S. Bandurski, "Indole as a precursor of indole-3-acetic acid in Zea mays”, Phytochemistry, 35(4), (1994), pp.905-909.

[12] T. Yakushi and K. Matsushita, "Alcohol dehydrogenase of acetic acid bacteria: structure, mode of action, and applications in biotechnology". Applied microbiology and biotechnology, 86(5), (2010), pp.1257-1265.

[13] C.M. Pazuch, D.L. Kalschne, F.B. Siepmann, I.M.G. Marx, T.C.G.D. Oliveira, W.A. Spinosa, C. Canan and E. Colla, "Optimization and characterization of vinegar produced from rice bran", Food Science and Technology, 40, (2019), pp.608-613.

[14] S. Miura, T. Arimura, N. Itoda, L. Dwiarti, J.B. Feng, C.H. Bin and M. Okabe, "Production of L-lactic acid from corncob”, Journal of bioscience and bioengineering, 97(3), (2004) pp.153-157.

[15] Z. Xu, Q. Wang, Z. Jiang, X.X. Yang and Y. Ji, "Enzymatic hydrolysis of pretreated soybean straw”, Biomass and Bioenergy, 31(2-3), (2007) pp.162-167.

[16] B. Schink, "Energetics of syntrophic cooperation in methanogenic degradation”, Microbiology and molecular biology reviews, 61(2), (1997), pp.262-280.

[17] D.A. Glassner and R. Datta, "Process for the production and purification of succinic acid”, Patent NumberUS Patent 5, 143, (1992), 834.

[18] N.S. Samuelov, R. Lamed, S. Lowe and J.G. Zeikus, "Influence of CO2-HCO3- levels and pH on growth, succinate production, and enzyme activities of Anaerobiospirillum succiniciproducens", Applied and environmental microbiology, 57(10), (1991), pp.30133019.

[19] Guettler, V. Michael and K. Mahendra Jain. "Method for making succinic acid, Anaerobiospirillum succiniciproducens variants for use in process and methods for obtaining variants", U.S. Patent No. 5,521,075. 28 May 1996.

[20] S.J. Lee, H. Song and S.Y.Lee, "Genome-based metabolic engineering of Mannheimia succiniciproducens for succinic acid production", Applied and environmental microbiology, 72(3), (2006), 1939-1948. 